EPJ Web of Conferences 23, 00021 (2012)

DOI: 10.1051/epjconf/20122300021

(C) Owned by the authors, published by EDP Sciences, 2012

\title{
Scanning Tunnelling Spectroscopic Studies of Dirac Fermions in Graphene and Topological Insulators
}

\author{
N.-C. Yeh ${ }^{1}$, M. L. Teague ${ }^{1}$, R. T.-P. Wu ${ }^{1}$, H. $\mathrm{Chu}^{2}$, D. A. Boyd ${ }^{2}$, M. W. Bockrath ${ }^{3}$, L. He ${ }^{4}$, F.-X. Xiu ${ }^{5}$ and K.-L. Wang \\ ${ }^{1}$ Department of Physics, California Institute of Technology, Pasadena, CA 91125, USA \\ ${ }^{2}$ Department of Applied Physics, California Institute of Technology, Pasadena, CA 91125, USA \\ ${ }^{3}$ Department of Physics and Astronomy, University of California, Riverside, CA 92521, USA \\ ${ }^{4}$ Department of Electrical Engineering, University of California, Los Angeles, CA 90095, USA \\ ${ }^{5}$ Department of Electrical and Computer Engineering, Iowa State University, Ames, IA 50011, USA
}

\begin{abstract}
We report novel properties derived from scanning tunnelling spectroscopic (STS) studies of Dirac fermions in graphene and the surface state (SS) of a strong topological insulator ( $\mathrm{STI}$ ), $\mathrm{Bi}_{2} \mathrm{Se}_{3}$. For mono-layer graphene grown on $\mathrm{Cu}$ by chemical vapour deposition (CVD), strain-induced scalar and gauge potentials are manifested by the charging effects and the tunnelling conductance peaks at quantized energies, respectively. Additionally, spontaneous time-reversal symmetry breaking is evidenced by the alternating anti-localization and localization spectra associated with the zero-mode of two sublattices while global time-reversal symmetry is preserved under the presence of pseudo-magnetic fields. For $\mathrm{Bi}_{2} \mathrm{Se}_{3}$ epitaxial films grown on $\mathrm{Si}(111)$ by molecular beam epitaxy (MBE), spatially localized unitary impurity resonances with sensitive dependence on the energy difference between the Fermi level and the Dirac point are observed for samples thicker than 6 quintuple layers (QL). These findings are characteristic of the SS of a STI and are direct manifestation of strong topological protection against impurities. For samples thinner than 6-QL, STS studies reveal the openup of an energy gap in the SS due to overlaps of wave functions between the surface and interface layers. Additionally, spin-preserving quasiparticle interference wave-vectors are observed, which are consistent with the Rashba-like spin-orbit splitting.
\end{abstract}

\section{Introduction - Dirac materials}

The Dirac materials are a new class of matter that exploits the mapping of electronic band structures and an embedded spin or pseudo-spin degree of freedom onto the relativistic Dirac equation [1-6]. These materials, including graphene [1] and the surface state (SS) of threedimensional (3D) strong topological insulators (STI) [26], have emerged as a new paradigm in condensed matter for investigating the topological phases of massless and massive Dirac fermions. Among the novel characteristics of massless Dirac materials, a common phenomenon is the topological protection of Dirac fermions against backscattering as the result of prohibition of inverting spin [2] or pseudo-spin [1] under time reversal operation due to the large spin-orbit or inter-valley interaction.

In this work, we report studies of massless Dirac fermions in two types of Dirac materials: the mono-layer graphene and the SS of a $3 \mathrm{D}-\mathrm{STI}, \mathrm{Bi}_{2} \mathrm{Se}_{3}$, by means of scanning tunnelling microscopy and spectroscopy (STM/STS). For mono-layer CVD-grown graphene on $\mathrm{Cu}$, we find spectroscopic evidences for strain-induced scalar and gauge potentials [7]. The gauge potentials result in both integer and fractional quantum Hall (IQH and $\mathrm{FQH}$ ) states, and the finding of $\mathrm{FQH}$ states is attributed to strong short-range Coulomb interaction mediated by the underlying $\mathrm{Cu}$ substrate. Moreover, alternating anti-localization and localization spectra associated with the zero-mode of the two sublattices are observed as the result of local spontaneous time-reversal symmetry breaking. For MBE-grown $\mathrm{Bi}_{2} \mathrm{Se}_{3}$ epitaxial films on $\mathrm{Si}(111)$ in the $3 \mathrm{D}$ limit, spatially localized unitary impurity resonances with sensitive dependence on the energy difference between the Fermi level $\left(E_{F}\right)$ and the Dirac point $\left(E_{D}\right)$ are demonstrated [8]. These findings are consistent with theoretical predictions for impurities in the SS of a STI $[9,10]$. In contrast, STS studies of samples in the 2D limit reveal the opening of a SS energy gap and the appearance of spin-preserving quasiparticle interference (QPI) wave-vectors.

\section{Evidence of strain-induced gauge and scalar potentials and spontaneous time- reversal symmetry breaking in graphene}


The electronic properties of graphene are known to be highly susceptible to the surrounding environment and disorder. In general, there are two primary effects associated with disorder on the electronic properties of graphene [1]. The first effect is a local change in the single site energy that leads to an effective shift in the chemical potential and thus scattering effects for Dirac fermions [1]. The second type of disorder effect arises from changes in the distance or angles between the $p_{z}$ orbitals [1]. In this case, the hopping energies between different lattice sites are modified, leading to the addition of a new term in the appearance of a vector (gauge) $\mathcal{A}$ and scalar potentials $\Phi$ to the original Dirac Hamiltonian [1]. The addition of a disorder-induced vector potential $\mathcal{A}$ in the Hamiltonian leads to the occurrence of an effective magnetic field $\boldsymbol{B}_{S}=\left(c / e v_{F}\right) \nabla \times \mathcal{A}$ for the Dirac cone at $\mathrm{K}$ and an opposite field $-\boldsymbol{B}_{S}=\left(c / e v_{F}\right) \nabla \times \mathcal{A}^{*}$ for the inequivalent Dirac cone at $\mathrm{K}^{\prime}$, where $v_{F}$ denotes the Fermi velocity of the Dirac fermions [11]. Therefore, the global time-reversal symmetry is preserved under the addition of such a gauge potential. Similarly, the presence of a spatially varying scalar potential can lead to local charging effects known as self-doping [1].

\subsection{Pseudo-magnetic fields, charge interactions, and integer \& fractional quantum Hall states}

\subsubsection{Strain-induced pseudo-magnetic fields}

One mechanism for inducing pseudo-magnetic fields and charging effects is strain [7,11-13]. Specifically, the strain-induced gauge potential $\mathcal{A}=\left(A_{x}, A_{y}\right)$ may be related to the $2 \mathrm{D}$ strain field $\mathbf{u}_{i j}(x, y)$ by the following relation (with $x$-axis along the zigzag direction) $[1,7]$ :

$$
\mathcal{A}=\frac{-(\partial \ln t / \partial \ln a)}{a}\left(\begin{array}{c}
u_{x x}-u_{y y} \\
-2 u_{x y}
\end{array}\right) \equiv \frac{\beta}{a}\left(\begin{array}{c}
u_{x x}-u_{y y} \\
-2 u_{x y}
\end{array}\right)
$$

where the variable $t$ denotes the nearest hopping constant, $a$ represents the nearest carbon-carbon distance, $\beta$ is a constant ranging from 2 to 3 [1], and the strain field $\mathbf{u}_{i j}(x, y)$ is related to a $2 \mathrm{D}$ displacement field $\mathbf{u}=\left(u_{x}, u_{y}\right)$ by the definitions $u_{x x} \equiv\left(\partial u_{x} / \partial x\right), u_{x y} \equiv\left(\partial u_{x} / \partial y\right)$ and $u_{y y} \equiv$ $\left(\partial u_{y} / \partial y\right)$. For non-trivial strain that leads to an effective magnetic field $\boldsymbol{B}_{S}=\left(c / e v_{F}\right) \nabla \times \mathcal{A}$, the local density of states (LDOS) of Dirac fermions is modified so that LDOS peaks at quantized energies $\left(E_{n}\right)$

$$
E_{n}=\operatorname{sgn}(n) \sqrt{2 e v_{F}^{2} \hbar B_{S}|n|}
$$

appear in addition to the linear density of states $\mathcal{N}(E)$ per unit cell of unstrained graphene, where

$$
\mathcal{N}(E)=\left(3 \sqrt{3} a^{2} / \pi\right)\left(E / v_{F}^{2}\right)
$$

In Eq. (2) $n$ denotes either integers or fractional numbers. Hence, strain-induced pseudo-magnetic fields $\boldsymbol{B}_{\boldsymbol{S}}$ may be inferred from the quantized tunneling conductance peaks in the STS studies of strained graphene by identifying the slope of the $\left(E_{n}-E_{D}\right)$ vs. $|n|^{1 / 2}$ plot, where $E_{D}$ is the Dirac energy [11].

In this work, we performed STM/STS studies of graphene grown by chemical vapor deposition (CVD) on $\mathrm{Cu}$ [7]. Details for the CVD growth process and STM/STS measurements have been given elsewhere [7]. We found that large and non-uniform strain was induced by the expansion of graphene and the contraction of the copper substrate upon cooling from the CVD-growth temperature $\left(\sim 1000^{\circ} \mathrm{C}\right)$ to low temperatures [7], which resulted in significant lattice distortions at atomic scales, as exemplified in figure 1(a). In addition, local variations in the Dirac energy $E_{D}$ are also present, as shown in figure 1(b) for the map of Dirac energies over the same area as shown in figure 1(a). (a)

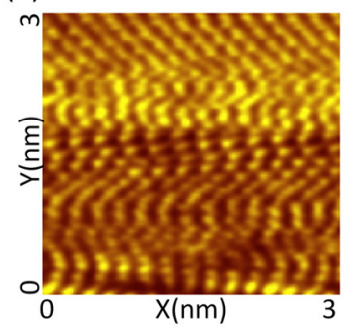

(b)

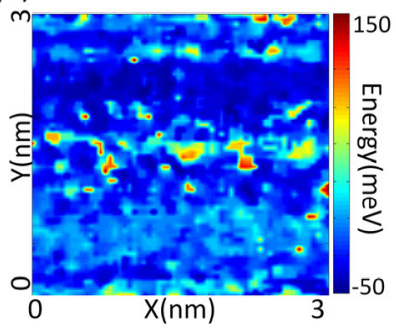

Fig. 1. Topographic and conductance studies of strained CVDgrown graphene on $\mathrm{Cu}$ : (a) Atomically resolved topography of graphene, showing strongly distorted lattices over a $(3 \times 3) \mathrm{nm}^{2}$ area. (b) Variations in the Dirac energy $E_{D}$ over the same area as shown in (a).

\subsubsection{Manifestation of two zero modes due to local spontaneous time-reversal symmetry breaking}

While the strain-induced pseudo-magnetic fields do not break global time-reversal symmetry, the opposite gauge fields associated with the two pseudo-spins (valleys) $\mathrm{K}$ and $\mathrm{K}^{\prime}$ in reciprocal space in fact give rise to a peculiar zero mode, which is a condensate where Dirac fermions become completely delocalized over the sample and yet remain alternately localized and anti-localized in the pseudo-spin projection in the real space [14]. More specifically, the presence of a pseudo-magnetic field due to a repulsive second-nearest-neighbor interaction, such as that provided by a triaxial strain in a graphene nanobubbles [13], can provide a net flux of a non-Abelian gauge field that preserves the time reversal symmetry and breaks the chiral symmetry of the free Dirac Hamiltonian. This non-Abelian gauge field is shown theoretically [14] to serve as a catalyst for local spontaneous breaking of the time-reversal symmetry in graphene while preserving the chiral symmetry.

Empirically, the local spontaneous time-reversal symmetry breaking may be manifested by the alternating presence and absence of the tunneling conductance peak at $n=0$ for two inequivalent sublattice sites in graphene. Indeed, our spatially resolved STS studies of the CVDgrown graphene on $\mathrm{Cu}$ revealed tunneling spectra with alternating presence and absence of the zero mode, as exemplified in figures $2(a)-(b)$. This finding is the first 
direct evidence of spontaneous time-reversal symmetry breaking due to strain-induced gauge fields in graphene.
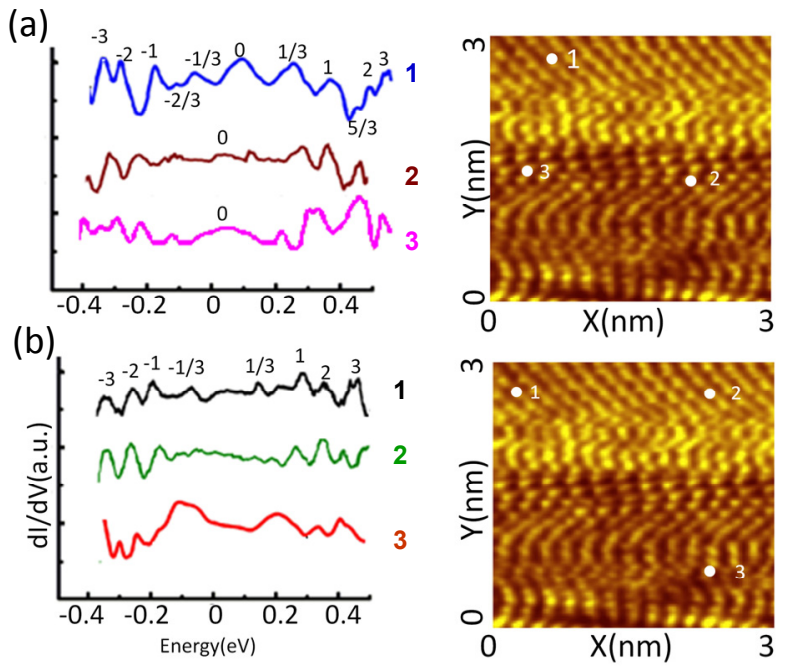

Fig. 2. Manifestation of strain-induced pseudo-magnetic fields in the tunnelling conductance $(d I / d V)$ versus $\left(E-E_{D}\right)$ spectra of CVD-grown graphene on $\mathrm{Cu}$ at $T=77 \mathrm{~K}$ : (a) Left panel, representative point spectra at A-sites after subtraction of the free Dirac fermion spectral background [7], showing distinct conductance peaks at integer and fractional quantized energies $E_{n}$ as defined in Eq. (2), where $E_{n}$ is proportional to $\pm|n|^{1 / 2}$. Peaks are clearly visible for $n=0, \pm 1, \pm 2, \pm 3$. Additional humps at fractional values $n=-5 / 3,-2 / 3$ and $\pm 1 / 3$ are also identifiable. Right panel, atomically resolved topography of the graphene showing the locations (represented by the white dots 1,2,3) where the zero mode is present. (b) Left panel, representative point spectra at B-sites after subtraction of the free Dirac fermion spectral background, showing distinct conductance peaks at $n= \pm 1 / 3, \pm 1, \pm 2, \pm 3$, with absence of zero mode at $n=$ 0 . Right panel, atomically resolved topography of the graphene showing the locations (represented by the white dots 1,2,3) where the zero mode is absent.

\subsubsection{Fractional quantum Hall (FQH) states due to interacting Dirac fermions}

To date the theoretical descriptions for strain-induced gauge potentials $[11,12]$ have assumed non-interacting Dirac fermions in graphene. Hence, only integer quantum Hall (IQH) states are expected under pseudo-magnetic fields. On the other hand, for CVD-grown graphene on $\mathrm{Cu}$, the 2D electron gas (2DEG) associated with the surface state of $\mathrm{Cu}$ substrates provides an additional channel for short-range charge interaction through the electrostatic coupling between the Dirac fermions in graphene and the 2DEG in $\mathrm{Cu}$. Although long-range Coulomb interactions of Dirac fermions are screened by the underlying 2DEG, whether short-range Coulomb interactions may be neglected depends on the strength of onsite Coulomb potential $U$ relative to the nearestneighbor hopping constant $t$. That is, if we consider the Hamiltonian

$\mathcal{H}=-t \sum_{\langle i, j\rangle, \sigma}\left(a_{i \sigma}^{\dagger} b_{j \sigma}+a_{i \sigma} b_{j \sigma}^{\dagger}\right)+U \sum_{i} n_{i \uparrow} n_{i \downarrow}$ where $a_{i \sigma}\left(a_{i \sigma}^{\dagger}\right)$ annihilates (creates) an electron with spin $\sigma(\sigma=\uparrow, \downarrow)$ on site $i$ of the sublattice A, $b_{j \sigma}\left(b_{j \sigma}^{\dagger}\right)$ annihilates (creates) an electron with spin $\sigma$ on site $j$ of the sublattice $\mathrm{B}, t \approx 2.8 \mathrm{eV}[1]$ is the nearest-neighbor hopping energy for hopping between different sublattices, and $n_{i \uparrow}$ and $n_{i \downarrow}$ denote the density of electrons with spin up and spin down at site $i$, respectively. Given the onsite Coulomb repulsion $U_{\mathrm{Cu}} \sim 3 \mathrm{eV}$ for the surface state of $\mathrm{Cu}$ [15], the onsite Coulomb potential $U$ for graphene in Eq. (4) is approximated by the relation $U=U_{\mathrm{Cu}}\left(d_{\mathrm{Cu}} / d\right)^{2}$, where $d_{\mathrm{Cu}} \sim 0.255 \mathrm{~nm}$ and $d=3^{1 / 2} a \sim 0.246 \mathrm{~nm}$ are the lattice constants of copper and graphene, respectively. Hence, we find $U \sim 3.2 \mathrm{eV}$, which is larger than the hopping constant $t$, implying that the Coulomb interaction among Dirac fermions cannot be neglected. Thus, fractional quantum Hall $(\mathrm{FQH})$ states become feasible, with the Laughlin state of a filling factor $v= \pm 1 / 3$ and the related states of $v= \pm 2 / 3, \pm 4 / 3$ and $\pm 5 / 3$ being the dominant contributions. This rationale is consistent with our experimental findings exemplified in figure 2 .

\subsection{Strained-induced scalar potential}

The strain found in the CVD-grown graphene on copper is not purely shear but also contains dilation/compression components, which is theoretically predicted to gives rise to an effective scalar potential $\Phi(x, y)$ and therefore a static charging effect $[12,16,17]$ in addition to the pseudo-magnetic field. Specifically, the scalar potential $\Phi(x, y)$ is given by $[12,16,17]$ :

$\Phi(x, y)=\varphi_{0}\left(u_{x x}+u_{y y}\right)=\varphi_{0}\langle|\mathbf{u}|\rangle$

where $\varphi_{0} \sim 3 \mathrm{eV}$, and $\langle|\mathbf{u}|\rangle$ is the dilation/compression strain. While the charging effect may be largely screened if the height variation $l$ is much smaller than the magnetic length $l_{B} \equiv\left[\Phi_{0} /\left(2 \pi B_{S}\right)\right]^{1 / 2}$, where $\Phi_{0}$ is the flux quantum [11,12], our finding of $B_{S}$ ranging from 30 to 50 Tesla corresponds to values of $l_{B}$ ranging from $5.5 \mathrm{~nm}$ to 3.5 $\mathrm{nm}$ [7], which is comparable to the height variation $l \sim 1$ $\mathrm{nm}$ over the sample area shown in figure 1(a). Hence, we expect significant charging effect in the CVD-grown graphene on $\mathrm{Cu}$, which may be manifested by the spatial variations in the constant-voltage tunneling conductance.

(a)

(b)
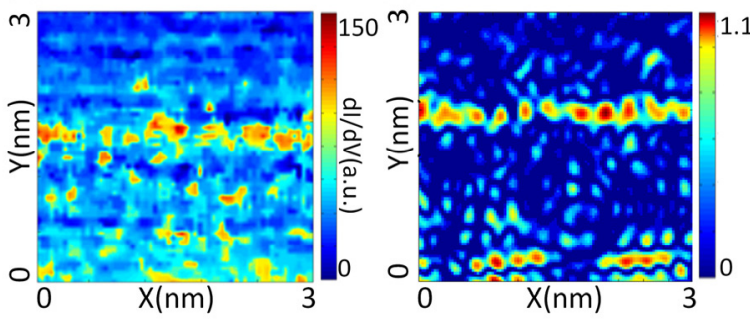

Fig. 3. Correlation of the compression/dilation strain and the charging effect is manifested by the $(3 \times 3) \mathrm{nm}^{2}$ spatial maps of (a) the zero-bias conductance, and (b) the compression/dilation strain, where the strain is estimated by considering the spatial variations in the height $z(x, y),\left[(\partial z / \partial x)^{2}+(\partial z / \partial y)^{2}\right]$. 
As shown in figures 3(a) and 3(b) for the spatial maps of the tunnelling conductance at zero bias and of the dilation/compression strain, respectively, there is clear correlation between the spatial distributions of the effective strain $\left[(\partial z / \partial x)^{2}+(\partial z / \partial y)^{2}\right]$ and the zero-bias conductance, where $z(x, y)$ denotes the spatial variations of the height. This apparent correlation thus provides direct evidence for the strain-induced charging effect.

\section{Impurity resonances and effects of dimensional crossover in the surface state of topological insulator $\mathrm{Bi}_{2} \mathrm{Se}_{3}$}

An exciting new development in condensed matter physics over the last decade is the beautiful realization of topological field theories [18] in strongly correlated electronic systems, where topological field theories are shown to provide a classification of order due to macroscopic entanglement that is independently of symmetry breaking [19]. The FQH state is the first known example of such a quantum state that exhibits no spontaneous broken symmetry, and its properties depend only on its topology rather than geometry, which is topologically distinct from all other quantum states classified by broken symmetry. Recently, the quantum spin Hall (QSH) states and the topological insulators (TI) have emerged as a new class of topological states and have stimulated intense research activities [2]. As described in Section 1, one of the novel properties associated with the TI is the presence of a Dirac spectrum of chiral low-energy excitations, which is a salient feature of the Dirac materials. In the case of 3D-STI, an odd number of massless Dirac cones in their SS are ensured by the $Z_{2}$ topological invariant of the fully gapped bulk [2-6]. Backscattering of Dirac fermions is suppressed due to topological protection that preserves the Dirac dispersion relation for any time-reversal invariant perturbation [2].

\subsection{Spectroscopic evidences for Fermi-energy dependent impurity resonances}

While direct backscattering is prohibited in both the SS of 3D-STI and in graphene, sharp resonances are not excluded because Dirac fermions with a finite parallel momentum may be confined by potential barriers [1]. In fact, theoretical calculations for Dirac fermions in the presence of non-interacting impurities have predicted the occurrence of strong impurity resonances $[9,10]$. Nonetheless, to date no direct empirical observation of strong resonances has been demonstrated despite reports of spectral evidences for quasiparticle interferences (QPI) associated with impurity or step-edge induced scattering [20-22] of Dirac fermions.

Here we report direct STS observation of impurity resonances in a $3 \mathrm{D}$-STI system, $\mathrm{Bi}_{2} \mathrm{Se}_{3}$ [8]. The samples that we investigated are epitaxial $\mathrm{Bi}_{2} \mathrm{Se}_{3}$ films grown on $\mathrm{Si}(111)$ by molecular beam epitaxy (MBE). Details of the MBE film growth process have been described elsewhere [23]. Transmission electron microscopy (TEM) on these films exhibited perfect triangular lattice structures, and
ARPES (angle resolved photoemission spectroscopy) studies revealed a single Dirac cone for the SS [23]. We performed STS studies [8] on samples of three average thicknesses: 60, 7 and 3 quintuple layers (QLs), because the energy difference $\left(E_{F}-E_{D}\right)$ of $\mathrm{MBE}$-grown $\mathrm{Bi}_{2} \mathrm{Se}_{3}$ epitaxial films was found to increase with decreasing thickness [24]. In particular, a crossover of the SS from a gapless Dirac cone to gapped spectra occurred when the average thickness became less than 6 QLs, and the energy gap further increased with decreasing thickness [24, 25].

Generally the spectra in our STS studies were found consistent throughout a large flat area [8]. Representative point spectra for the 60-QL and 7-QL samples are given in the main panels of figures 4(a) and 4(b), and the ranges of the Dirac energy $E_{D}$ for all areas investigated are summarized in the insets of figures 4(a) and 4(b), which correspond to $E_{D}=(-78 \pm 38) \mathrm{meV}$ and $E_{D}=(-100 \pm 25)$ $\mathrm{meV}$, respectively.
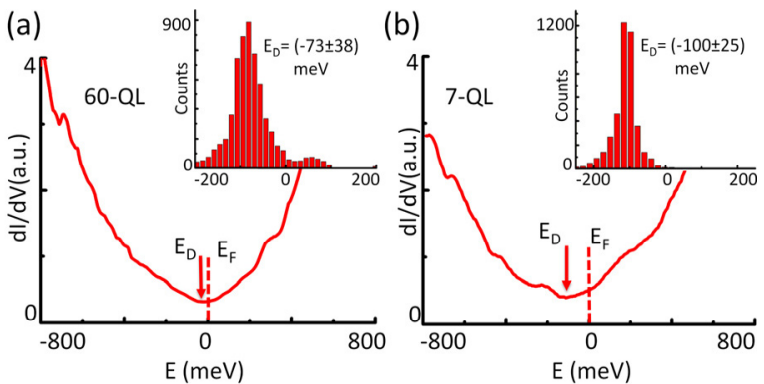

Fig. 4. Representative tunnelling conductance spectra (main panel) of MBE-grown $\mathrm{Bi}_{2} \mathrm{Se}_{3}$ epitaxial films of (a) $60-\mathrm{QL}$ and (b) 7-QL thickness. The insets are histograms of the Dirac energy $E_{D}$ over extended areas of the 60-QL and 7-QL samples, showing $E_{D}=(-78 \pm 38) \mathrm{meV}$ for $60-\mathrm{QL}$ and $E_{D}=(-100 \pm 25)$ $\mathrm{meV}$ for 7-QL. We further note that in STS studies the Fermi energy $E_{F}$ coincides with the zero energy.

Despite the relatively consistent tunneling spectra for most areas investigated, we note the presence of a few quantum impurities, as exemplified by the high conductance spots in figure 5(a) for the constant-bias conductance map of a 60 -QL sample over a $(5.0 \times 8.0)$ $\mathrm{nm}^{2}$ area and in figure 5(b) for a 7-QL sample over a (5.1 $\times 8.8) \mathrm{nm}^{2}$ area. The tunneling spectra on top of the quantum impurities are exemplified in figure 6(b) for the 60-QL and 7-QL samples.

There are several noteworthy spectral characteristics for the quantum impurities. First, sharp conductance peaks associated with the presence of impurities, $\Omega_{\text {imp }}$, generally occur at energies near the Dirac point $E_{D}$, which are consistent with the unitary limit of impurity scattering so that the impurity potential $U_{\text {imp }}$ diverges following the relation [17]

$\left(\Omega_{\text {imp }}-E_{D}\right) \sim 5 \operatorname{sgn}\left(U_{\text {imp }}\right) /\left(\left|U_{\text {imp }}\right| \ln \left|U_{\text {imp }}\right|\right)$.

Second, the conductance peaks are strongly localized in space, within a radius of $\sim 0.2 \mathrm{~nm}$ around each impurity, implying rapid recovery of the SS of Dirac fermions away from the impurities. Third, the intensity of the 
impurity resonances decreases rapidly with the increasing energy difference $\left|E_{F}-E_{D}\right|$.

The rapidly diminishing spectral intensity of impurity resonances with increasing $\left|E_{F}-E_{D}\right|$ is consistent with the theory for non-interacting point impurities embedded in a system of massless Dirac fermions [10]. Following the theoretical analysis in reference [10] that utilized the Keldysh Green function formalism, we showed in reference [8] and reproduced in figure 6(a) the theoretical dependence of the intensity of impurity resonances on $\left(E_{F}\right.$ $\left.-E_{D}\right)$, which is in reasonable agreement with our empirical findings [8]. (a)

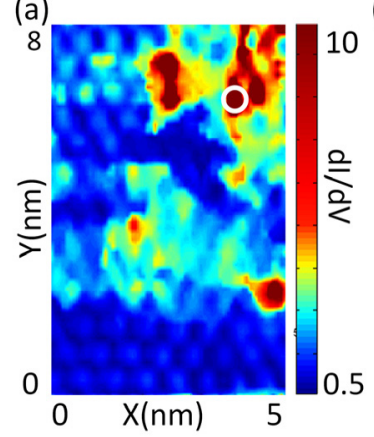

(b)

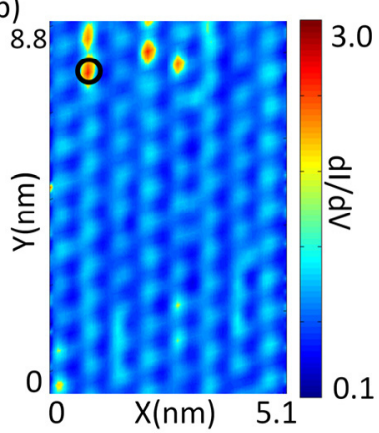

Fig. 5. Representative atomically resolved constant energy $(E)$ conductance maps of MBE-grown $\mathrm{Bi}_{2} \mathrm{Se}_{3}$ epitaxial films of (a) 60-QL taken at $E=-30 \mathrm{meV}$ and (b) 7-QL taken at $E=-50$ $\mathrm{meV}$. The high conductance spots are associated with quantum impurities embedded in the atomically ordered $\mathrm{Bi}_{2} \mathrm{Se}_{3}$ host, and the intensity of the impurity resonance is found to be highly localized so that the generic spectrum for the SS of the 3D-STI recovers within $\sim 0.2 \mathrm{~nm}[8]$. The point spectrum at the location indicated by the white circle in (a) and that at the location indicated by the black circle in (b) are given in figure 6(b), showing sharp resonant peaks near the respective Dirac energies $E_{D}$, implying that the impurity potential is in the unitary limit [9]. (a)

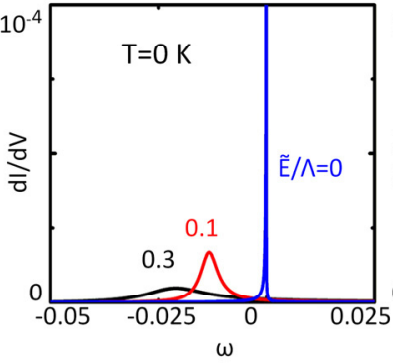

(b)

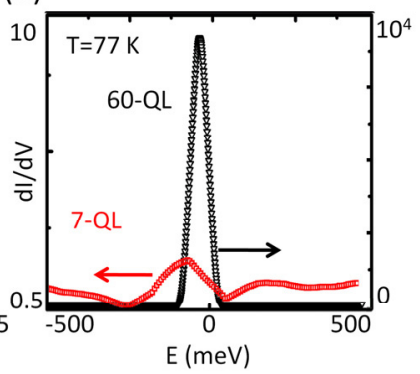

Fig. 6. (a) Theoretical predictions for the dependence of the intensity of impurity resonances on $(\tilde{E} / \Lambda)$, where $\Lambda$ denotes a cutoff energy and $\tilde{E} \equiv\left(E_{F}-E_{D}\right)[8,10]$. (b) Representative point spectra above the quantum impurities in the $60-\mathrm{QL}$ and 7QL samples, showing strong resonances at energies very close to $E_{D}$. The spectrum for a point impurity of the 60-QL sample was taken at the location indicated by a white circle in figure 5(a), and that for a point impurity of the 7-QL sample was taken at the location denoted by a black circle in figure 5(b).

\subsection{Effects of dimensional crossover}

While the dispersion relation of the SS of 3D-STI has been confirmed to be a gapless Dirac cone, for 3D-STI in the thin-film limit the coupling between opposite topological surface states in space is theoretically expected to open up an energy gap [25]. Therefore, the dispersion relation will convert from a Dirac cone into a gapped Dirac hyperbola [25]. The thin film may further break the top-bottom symmetry due to the asymmetric chemical potentials between the surface and the interface of the thin film with a substrate, leading to a Rashba-like coupling and further energy splitting in the momentum space [25]. These theoretical predictions have been verified by $A R P E S$ studies of MBE-grown $\mathrm{Bi}_{2} \mathrm{Se}_{3}$ films on $\mathrm{SiC}$ [24], which demonstrated that the surface spectra open a visible energy gap when the thickness is below 6 QLs. The energy gap was found to increase with decreasing thickness in thin films of 2-6 QL.

(a)

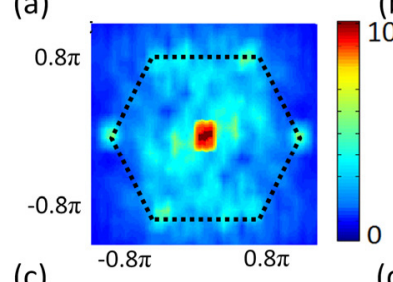

(c)

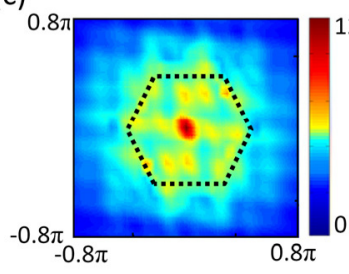

(b)

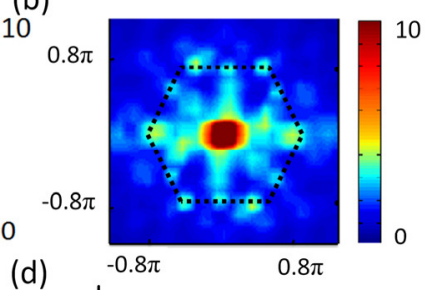

(d)

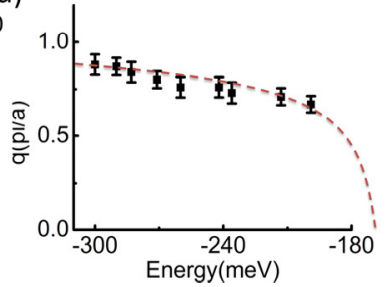

Fig. 7. Evidence for quasiparticle interferences (QPI) in the 5QL $\mathrm{Bi}_{2} \mathrm{Se}_{3}$ sample from Fourier transformed (FT) tunneling conductance maps that reveal energy $(E)$ dependent QPI wavevectors $(q)$ with preservation of spin texture, where the $q$-value for each FT-conductance map is obtained by the wave-vector from the center of the zone to the one of the 6 points on the hexagon: (a) $E=-300 \mathrm{meV}$, (b) $E=-260 \mathrm{meV}$, and (c) $E=$ $-80 \mathrm{meV}$. The energy dependence of the QPI wave-vectors $q(E)$ determined from all FT conductance maps is summarized in (d), showing decreasing $|\mathbf{q}|$ values as $E$ approaches $E_{D}$.

A natural consequence of the Rashba-like coupling and the gapped Dirac hyperbola in the thin-film limit of a 3D-STI is that the resulting energy dispersion relation would involve four split Fermi surfaces with chiral spin textures for surface-state spin-up, interface-state spin-up, surface-state spin-down, and interface-state spin-down [25]. Therefore, quasiparticles interferences (QPI) due to elastic impurity scattering are expected only between Fermi surfaces with the same spin texture, similar to the findings from STS studies of the QPI in $\mathrm{Bi}_{x} \mathrm{Sb}_{1-x}$ [20]. However, we note that $\mathrm{Bi}_{x} \mathrm{Sb}_{1-x}$ is a $3 \mathrm{D}$-STI with much more complicated Fermi surfaces and therefore multiple spin-preserved QPI wave-vectors are allowed [20]. In contrast, for the four split Fermi surfaces in $\mathrm{Bi}_{2} \mathrm{Se}_{3}$ thin 
films, only one value for the QPI wave-vector $\mathbf{q}$ is expected for a given quasiparticle energy due to the preservation of spin textures from spin-up to spin-up states and from spin-down to spin-down states. Further, the $|\mathbf{q}|$-values should be smaller than the reciprocal lattice constants, and should decrease as $E \rightarrow E_{D}$.

Our preliminary STS studies of a $5-\mathrm{QL} \mathrm{Bi}_{2} \mathrm{Se}_{3}$ thin film on $\mathrm{Si}$ (111) revealed that the Fourier-transformed (FT) conductance maps at various constant energies indeed exhibited a single QPI $|\mathbf{q}|$-value for a given energy, as exemplified in figures $7(\mathrm{a})-7(\mathrm{c})$. Further, the magnitude of QPI wave-vectors indeed decreases with quasiparticle energy $E$ approaching the Dirac point $E_{D}$, as shown in figure 7(d). These results are qualitatively consistent with preservation of spin textures in QPI for 3D-STI in the thin-film limit. However, detailed analysis of our STS data is not in quantitative agreement with the fitting parameters derived from ARPES studies of $\mathrm{Bi}_{2} \mathrm{Se}_{3}$ films on $\mathrm{SiC}$ [24]. Possible reasons for the occurrence of discrepancies may include different substrates used, different $\left(E_{F}-E_{D}\right)$ values for each nominal sample thickness $[8,24]$, and variations in the physical properties from microscopic to macroscopic experiments. Further systematic STS studies of $\mathrm{Bi}_{2} \mathrm{Se}_{3}$ films with different thicknesses and substrates will be necessary to resolve the quantitative discrepancies.

\section{Conclusion}

We report in this work scanning tunnelling spectroscopic (STS) studies of Dirac fermions in graphene and the surface state (SS) of a three-dimensional (3D) strong topological insulator (STI), $\mathrm{Bi}_{2} \mathrm{Se}_{3}$. In the case of CVDgrown graphene on $\mathrm{Cu}$, we find that strain-induced scalar and gauge potentials are manifested by the charging effects and the tunnelling conductance peaks at quantized energies, respectively. The observation of both integer and fractional quantum Hall $(\mathrm{IQH} / \mathrm{FQH})$ states due to strain-induced pseudo-magnetic fields may be attributed to significant short-range Coulomb interactions of Dirac fermions in graphene mediated by the underlying $\mathrm{Cu}$ substrate, so that the resulting Coulomb interaction $U \sim$ $3.2 \mathrm{eV}$ is larger than the nearest-neighbour hopping energy $t \sim 2.8 \mathrm{eV}$ in graphene. Further, spontaneous timereversal symmetry breaking is evidenced by the spatially alternating presence and absence of the pseudo-magnetic field-induced zero mode in the tunnelling conductance spectra while global time-reversal symmetry is preserved.

For MBE-grown $\mathrm{Bi}_{2} \mathrm{Se}_{3}$ epitaxial films on $\mathrm{Si}(111)$, unitary impurity resonances with sensitive dependence on the energy difference between the Fermi level and the Dirac point are observed for samples thicker than 6 quintuple layers (QL). These resonances are spatially localized within an area of radius $\sim 0.2 \mathrm{~nm}$, implying strong topological protection against impurities. For samples thinner than 6-QL, STS studies reveal the openup of an energy gap in the SS due to overlaps of wave functions between the surface and interface layers and Rashba-like spin-orbit splitting. Additionally, spinpreserving quasiparticle interference (QPI) wave-vectors are observed as a function of quasiparticle energy, which are qualitatively consistent with the dimensional crossover behaviour of 3D-STI.

\section{Acknowledgement}

The research on graphene at Caltech was supported by the National Science Foundation, and the research on topological insulators at Caltech and UCLA was supported by the Center on Functional Engineered Nano Architectonics (FENA).

\section{References}

1. A.H. Castro Neto, F. Guinea, N.M.R. Peres, K.S. Novoselov, A.K. Geim, Rev. Mod. Phys. 81, 109 (2009).

2. X.-L. Qi and S.-C. Zhang, Phys. Today 63, 33 (2010).

3. L. Fu and C. L. Kane, Phys. Rev. B 76, 045302 (2007).

4. H. Zhang, C.-X. Liu, X.-L. Qi, X. Dai, Z. Fang and S.-C. Zhang, Nat. Phys. 5, 438 (2009).

5. J. E. Moore and L. Balents, Phys. Rev. B 75, 121306 (2007).

6. A. P. Schnyder, S. Ryu, A. Furusaki, and A. W. W. Ludwig, Phys. Rev. B 78, 195125 (2008).

7. N.-C. Yeh, M. L. Teague, S. Yeom, B. L. Standley, R. T.-P. Wu, D. A. Boyd, M. W. Bockrath, Surf. Sci. 605, 1649 (2011).

8. M. L. Teague, H. Chu, F.-X. Xiu, L. He, K.-L. Wang and N.-C. Yeh, submitted to Europhys. Lett. (2011).

9. R. R. Biswas and A. V. Balatsky, Phys. Rev. B 81, 233405 (2010).

10. K. Saha, I. Paul and K. Sengupta, Phys. Rev. B 81, 165446 (2010).

11. F. Guinea, M.I. Katsnelson, A.K. Geim, Nat. Phys. 6, 30 (2010).

12. F. Guinea, M. I. Katsnelson, M. A. H. Vozmediano, Phys. Rev. B 77, 075422 (2008).

13. N. Levy, S. A. Burke, K. L. Meaker, M. Panlasigui, A. Zettl, F. Guinea, A. H. Castro Neto, M. F. Crommie, Science 329, 544 (2010).

14. I. F. Herbut, Phys. Rev. B 78, 205433 (2008).

15. I. V. Solovyev, A. I. Liechtenstein and K. Terakura, Phys. Rev. Lett. 80, 5758 (1998).

16. H. Suzuura and T. Ando, Phys. Rev. B 65, 235412 (2002).

17. J. L. Manes, Phys. Rev. B 76, 045430 (2007).

18. D. Birmingham, M. Blau, M. Rakowski and G. Thompson, Phys. Rep. 209, 129 (1991).

19. X.-G. Wen, Adv. Phys. 44, 405 (1995).

20. P. Roushan, J. Seo, C. V. Parker, Y. S. Hor, D. Hsieh, D. Qian, A. Richardella, M. Z. Hasan, R. J. Cava and A. Yazdani, Nature 460, 1106 (2009).

21. T. Zhang, P. Cheng, X. Chen, J.-F. Jia, X. Ma, K. He, L. Wang, H. Zhang, X. Dai, Z. Fang, X. Xie, and Q.-K. Xue, Phys. Rev. Lett. 103, 266803 (2009).

22. Z. Alpichshev, J. G. Analytis, J.-H. Chu, I. R. Fisher, Y. L. Chen, Z. X. Shen, A. Fang, and A. Kapitulnik, Phys. Rev. Lett. 104, 016401 (2010). 
23. L. He, F.-X. Xiu, Y. Wang, A. V. Fedorov, G. Huang, X. Kou, M. Lang, W. P. Beyermann, J. Zou, and K.-L. Wang, J. Appl. Phys. 109, 103702 (2011).

24. Y. Zhang, K. He, C.-Z. Chang, C.-L. Song, L.-L. Wang, X. Chen, J.-F. Jia, Z. Fang, X. Dai, W.-Y. Shan, S.-Q. Shen, Q. Niu, X.-L. Qi, S.-C. Zhang, X.C. Ma and Q.-K. Xue, Nat. Phys. 6, 584 (2010).

25. W. Y. Shan, H.-Z. Lu and S.-Q. Shen, New J. Phys. 12, 043048 (2010). 